\title{
FLOR GARITA HERNÁNDEZ: SU CONTRIBUCIÓN A LA INVESTIGACIÓN Y A LA NORMALIZACIÓN TOPONÍMICA
}

\author{
Flor Garita Hernández: Her contribution to the investigation and \\ toponymic standardization
}

\section{Eduardo Bedoya Benitez ${ }^{*}$}

\begin{abstract}
RESUMEN
Texto de la participación en el Conversatorio que trata de la oficial tarea de normalización de nombres geográficos y en la investigación toponímica de la Sra. Flor Garita Hernández, profesora e investigadora homenajeada en la IV Semana de la Diversidad Lingüística, UCR, 5 de mayo, 2017.

Palabras clave: Toponimia, nomenclatura, nombres geográficos, investigación toponímica, Comisión Nacional de Nomenclatura.
\end{abstract}

\section{ABSTRACT}

Text of the participation in the Conversation that deals with the official task of normalization of geographical names and in the toponymic research of Mrs. Flor Garita Hernández, professor and researcher honored at the IV Semana de la Diversidad Lingüística, UCR, May 5, 2017.

Key Words: Toponymy, nomenclature, geographical names, toponymic research, National Commission of Nomenclature.

\section{Introducción: Feliz coincidencia}

En los años de 1972 y 1973 doña Flor Garita Hernández fue la profesora de español y literatura que guiaba una vez más a los cuartos y quintos años del área de letras en el Instituto Dr. Clodomiro Picado T., Turrialba. Mi objetivo de joven colegial era aprobar el quinto año y superar las pruebas de bachillerato. Ese fue mi primer contacto con ella, de simpatía a la distancia.

Entre los años 1975 y 1980 logré ver a la profesora acortando distancias por senderos en la ciudad universitaria Rodrigo Facio de Universidad de Costa Rica en San José. Fue hasta principios de 1990 cuando se organizó una reunión formal con una de las nuevas representantes de la Universidad de Costa Rica en la Comisión Nacional de Nomenclatura, esto con el director del Instituto Geográfico Nacional, Ing. Fernando Mauro Rudin Rodríguez, y mi persona como jefe del Departamento de División Territorial y Nomenclatura del mismo instituto, con la grata sorpresa de encontrarme con mi estimada profesora de secundaria exponiendo sobre el proyecto de investigación

\footnotetext{
* Universidad de Costa Rica. Profesor jubilado de la Escuela de Geografía. Costa Rica. Correo electrónico: jebb1956@gmail.com

Recepción: 06/02/2018. Aceptación: 01/03/2018.
} 
toponímica a nivel nacional que ella iniciaría en la provincia de Cartago. Ahí escuché por primera vez sobre el Modelo de Clasificación Ontogenético. El proyecto investigativo requería de cartografía (hojas topográficas), transporte (vehículo y operadores) y asistentes geógrafos para realizar las giras a campo, de lo que se dispuso de inmediato en facilitar. Desde ahí hubo una relación constante, tanto en las sesiones de la Comisión Nacional de Nomenclatura (CNN) como en las giras a campo aplicando entrevistas. Así comenzó también una relación de investigación y una gran amistad.

\section{Participación en la normalización de nombres geográficos}

\subsection{Contexto mundial de la normalización toponímica}

Con el desarrollo de los procesos que involucran a la totalidad del globo terráqueo, evidente con el desarrollo de la Primera Guerra Mundial y los efectos posteriores, se hizo forzosa la normalización toponímica, dada la convergente necesidad de todas las comunidades del planeta.

Los primeros atisbos de este interés mundial acontecieron en el Primer Congreso Geográfico Internacional, en 1871, cuando algunos de sus miembros presentaron mociones para lograr la "uniformidad" en la escritura de nombres que figuran en cartas, mapas e informes geográficos. Fue ya en el Congreso Geográfico Internacional de Ginebra, en 1908, en que se adoptaron formas para normalizar nombres geográficos en la producción de un mapa internacional a escala 1:1 000000 (Morandi, 2001, p. 72).

Este esfuerzo empezó a sistematizarse desde la Organización de las Naciones Unidas (ONU), a la vez iniciativas desde organismos continentales como la Organización de los Estados Americanos (OEA), entidades especializadas, como el Instituto Panamericano de Geografía e Historia (IPGH), y otros con intereses muy particulares por un orden mundial en materia de las denominaciones, como sucede con la Organización Internacional de Aviación Civil (OIAC).

\subsection{La Comisión Nacional de Nomenclatura}

La importancia dada a esta temática por estos organismos hizo eco también en organismos tales como los Institutos Geográficos de Centroamérica y Panamá, para que en eventos como las reuniones regionales, conocidas como las Semanas Cartográficas, 
se sugiriera promover la creación de organismos que normaran los nombres geográficos. No es casual que en Costa Rica se acataran y cumplieran de inmediato estas recomendaciones para regular la actividad toponímica, al extremo de darle carácter de ley de la República, la 3535 del 3 de agosto de 1965, que crea la Comisión Nacional de Nomenclatura. Con la trascripción de su primer artículo participamos de lo esencial:

Artículo $1^{\circ}$.- Crease una comisión encargada de velar porque los edificios y parajes públicos tengan nombres que constituyan homenaje a personas o sucesos de trascendencia histórica, social o cultural, y de preservar los nombres tradicionales y autóctonos de la geografía costarricense; a ese efecto procurará que no se produzca duplicidad en la nomenclatura, y tratará que desaparezca dentro de lo posible, las repeticiones que exista.

En todos los países del mundo existen responsables que abordan en alguna medida lo relativo a los nombres geográficos; estas organizaciones en esencia lo que pretenden al interior de cada país, estado o región de jurisdicción o administración es básicamente normalizar los nombres geográficos, sea la aplicación de una formalidad de aprobación del nombre que oficialmente llevará por ejemplo un río, un cerro o un determinado pueblo. Por lo general, aplican en:

Establecer y dar a conocer las normas y criterios para la normalización de los accidentes geográficos.

Efectuar el inventario de los nombres geográficos.

Realizar la normalización de los nombres geográficos de acuerdo con las normas establecidas.

Llevar un registro de los nombres geográficos.

Difundir la nomenclatura oficial

Asesorar en la materia de su especialización.

Incorporar dentro de sus atribuciones, lo que sobre la materia se resuelva en las reuniones regionales o internacionales y divulgar estas resoluciones (Rivas, 1986, p. 26).

En Costa Rica estas generalidades son parte del quehacer de la Comisión Nacional de Nomenclatura e incluso va más allá porque regula y norma los nombres de 
las nuevas obras públicas, así como parajes públicos. Para el 2017 esta Comisión cumplió 52 años de existencia y una amplia labor la respalda.

La comisión está adscrita al Ministerio de Cultura y Juventud, es conformada por miembros propietarios y suplentes que representan a este ministerio, al Instituto Geográfico Nacional, al Museo Nacional, a la Academia de Geografía e Historia de Costa Rica, a la Universidad Nacional y a la Universidad de Costa Rica.

Doña Flor Garita Hernández fue la representante de la UCR en la CNN desde 1990 al 2004, cuya labor generó un gran aporte en toda la demanda que requiere tal designación, con su presencia y la disciplina que le caracteriza, su aporte desde su profesión académica siempre fue a la altura, como el de sus antecesores (recordando con respeto y simpatía a doña Virginia Sandoval de Fonseca, doña Virginia Zúñiga Tristán, doña María Amoretti Hurtado, don Alfonso Cónstenla Umaña y don Víctor Sánchez Corrales). Trasciende el aporte de doña Flor Garita por las modificaciones a nivel de decreto en el articulado del Reglamento a la Ley 3535, así como aplicarla seria y constantemente en la investigación sistemática de la toponímica costarricense.

\subsection{La investigación toponímica}

\subsubsection{Qué y quién investiga la toponimia}

La toponimia apunta al análisis y el significado de los nombres propios que denominan lugares. Esta disciplina forma parte de la onomástica, la especialización de la lexicografía centrada en los nombres propios.

El perfil que encaja con el investigador en toponimia se sustenta o comprende básicamente tres ciencias: la lingüística, la historia y la geografía. Ernesto Querol califica a estas disciplinas como ciencias auxiliares de la toponimia (1995, pp. 64-65); igualmente lo afirma Jean Poirier (1965), citado por Joan Tort (2003), quien además agrega disciplinas como la psicología y la sociología. Pero la lista puede ampliarse con otras disciplinas cuando se acude a explicar relaciones con los nombres geográficos, tales como la dialectología, la fonética, la epigrafía, la arqueología, la archivística, la paleografía, entre otras.

La toponimia estudia un material que contiene una gran pluralidad de informaciones, de tal modo que puede haber innumerables formas de acceder a ellas y ninguna de ellas debe postergar a las otras; sólo así puede quedar la 
puerta abierta a hipótesis que no se han planteado pero que pueden tener su razón de ser en el futuro (Querol, 1995, p. 65).

La toponimia, como rama de la onomástica, es el estudio de los nombres de lugares. Según Hubschmid (1959), la toponimia tiene como objeto el estudio de la génesis de los nombres geográficos en su más amplio sentido: los odónimos, orónimos, hidrónimos, litónimos y otros grupos de nombres del terreno. Así, la toponimia es considerada como una ciencia humana. Unas disciplinas, o propiamente sus especialistas, la atraen a su alero. Algunos geógrafos la califican como una ciencia espacial, no solo por el simple hecho de figurar el topónimo como un elemento fundamental y propio en la cartografía, sino también por el hecho de ser o guardar un significado o ser descriptor del punto, área y accidente geográfico. El topónimo nos podría hablar de una posición, de una escritura de una lengua determinada, de los intercambios entre culturas, de su recorrido en el espacio -en el planeta y fuera de él-, del entorno - pasado y presente-, de las características del accidente y otros temas.

Esto es toponimia, esto es geografía, y si se practica desde otro alero científico, es aún mayor el enriquecimiento de las disciplinas.

\subsubsection{La investigación toponímica de Flor Garita}

Las investigaciones propias de la filóloga y lingüista Flor Garita Hernández son “Toponimia de la provincia de Cartago (1995), "Toponimia indígena de la provincia de Guanacaste" (1997), “Toponimia de la provincia de Heredia” (1997), “Toponimia de la provincia de Guanacaste” (2000), “Toponimia de la provincia de Puntarenas” (2002), “Toponimia de la provincia de Alajuela" (2010), "Toponimia de la red vial de la Gran Área Metropolitana" (2015). A esto deben añadirse las investigaciones que ha dirigido para el complemento del proyecto nacional de la toponimia de Costa Rica, trabajos que marcan una ardua tarea de casi tres décadas. Anteponiendo e interesándonos en el tema específico, esta labor nos habla de la tenacidad y la persistencia de una mujer que admiramos.

La principal contribución de doña Flor Garita Hernández es delinear un modelo básico de clasificación toponímica con un fundamento tal, en lo teórico, que permite su aplicación de manera universal. La autoría la comparte con don Adolfo Constenla Umaña. 
Los topónimos requieren de investigación de gabinete y campo para ser incorporados correctamente en el modelo; el resultado es la clasificación o taxonomía toponímica de todo un espacio geográfico mediante las fuentes escritas -libros, archivos y mapas-, y la recolección in situ con la entrevista de los vecinos de los lugares donde se sitúan los topónimos dudosos. Esto es otro aporte investigativo importante, pues nunca antes se había realizado un recorrido a escala provincial primero para sumarse, luego, a escala nacional. Los relatos populares constituyen explicaciones y anécdotas del origen y significado de los nombres geográficos, aporte al folclor o fuente para su análisis futuro.

Desde su primera aplicación, en la provincia de Cartago, doña Flor Garita establece que el modelo ha verificado los mecanismos por los que establece la relación lengua-cultura en la formación de los topónimos; los hábitos lingüísticos en la designación de topónimos se ponen a flote.

En Costa Rica se da la preferencia por el uso de vocablos construidos de elementos comunes del ambiente contra nombres propios preexistentes. De predilección por el sustantivo simple singular cuando se trata de la forma lingüística, con predominio de la sufijación de abundanciales en las provincias de Cartago, Alajuela y Guanacaste, más en la provincia de Heredia las construcciones con sufijos diminutivos. En la provincia de Puntarenas la preferencia del hablante es por el sustantivo simple, singulares o plurales, la forma simple en los orónimos, pero en los ecónimos predominan las frases específicas sobre el resto de estructuras, al denominar un sitio poblado lo hacen con un sustantivo y su respectivo calificativo.

En la investigación específica de la odonimia, doña Flor Garita tiene los mismos resultados, se enfatiza en la preferencia del hablante costarricense por las estructuras simples: sustantivos, con predominio en los singulares, adjetivos o frases sencillas. En cuanto a la morfología, el significado abundancial constituye la mayoría.

En la relación lengua-cultura, doña Flor Garita concluye que, a nivel nacional, el componente hispánico se impone al prehispánico. En nombres donde se tiene la certeza de su origen se encuentra la preferencia por los relacionados con árboles y plantas, individual o bien abundanciales o colectivos. 
En los nombres propios, los hagiónimos ocupan un lugar importante, esto equilibrado solamente en la provincia de Puntarenas. En Alajuela los hagiónimos son más frecuentes.

En Costa Rica hay predominio por los topónimos referidos a santos y no a santas. Se destaca particularmente “Ángeles" como homenaje a la Virgen, por la razón sobrada de ser la Patrona de Costa Rica.

En todo el país los antropónimos obedecen más a los apellidos. El concepto de familia como núcleo de la sociedad y el concepto de propiedad privada sobre todo en Guanacaste donde hubo y hay aún grandes haciendas.

Entre los nombres de pila los femeninos son mayoría, ya que la fundación de grupos habitacionales se les denomina como cumplido a la mujer de agrado, sea su esposa, sus hija o su madre; también en muchos casos por el mérito de ser las féminas las que forjaron con su trabajo la fundación de las fincas, caseríos y pueblos.

En los nombres relacionados con la red vial costarricense la situación cambia. Doña Flor Garita nos dice que los nombres propios son mayoría, lo que no ocurre con los ecónimos, hidrónimos y orónimos en las provincias. Al parecer las vías prestan o se facilitan más para el homenaje a personas, una cuestión urbana. Así los hagiónimos son minoría. Los nombres indígenas no tienen representación en esta clase de topónimo.

El entorno fundamenta la escogencia de los ecónimos, ya que los accidentes hídricos y los topográficos dan nombre a los centros poblados. Aún así, la flora prevalece con fuerte influencia en los ecónimos, y los de la fauna para los orónimos. En la provincia de Alajuela los nombres relacionados con la flora son mayoría en ecónimos, hidrónimos y orónimos. En fin, es notoria la biodiversidad del trópico y que se revierte irresistiblemente en la toponimia. Esto específicamente no ocurre en los odónimos, en los que se destacan las características del accidente como tal o del aporte humano al ambiente.

Doña Flor Garita agrega que en la relación lengua-cultura en Costa Rica hay un fuerte componente de nombres foráneos que justifican su existencia o relación con los primeros inmigrantes que se establecieron o por la admiración de los primeros pobladores hacia lugares más allá de las fronteras costarricenses.

Los resultados en investigaciones como "Toponimia de la provincia de San José”, de Rocío Monge y otras (2003), “Toponimia de la provincia de Limón”, de 
Guiselle Chang (2010), "Toponimia de las urbanizaciones de la Gran Área Metropolitana (GAM) de Costa Rica, de Yamilet Garbanzo y otras (2012) ratificaron la aplicación del modelo, sea su universalidad para determinar los hábitos toponímicos de los hablantes. Sus resultados prácticamente son similares a los alcanzados en las otras unidades administrativas en Costa Rica.

\section{Conclusión}

Mi formación como geógrafo profesional y el compromiso particular con la temática sirvió de complemento y soporte perfecto a doña Flor Garita, así mantenernos en constante investigación durante los últimos veinticinco años, hecho que corrobora la interdisciplinaridad de la toponimia. Esta coincidencia fue fundamental para iniciar un verdadero equipo de trabajo que ha fructificado en varias publicaciones sobre el tema. Pendiente está y hemos iniciado la toponimia de la isla del Coco, existen allá ochenta nombres geográficos que esperan de la aplicación del Modelo Ontogenético.

La experiencia que se iniciara con la investigación de la provincia de Cartago se continuaría al aplicar el mismo método y modelo en las otras seis provincias del país, además del análisis de los nombres de las calles y carreteras de la Gran Área Metropolitana. Hemos recorrido de norte a sur y de este a oeste nuestro territorio, lo que nos ha permitido conocer las diversas manifestaciones culturales del país.

La simbiosis lingüista-geógrafo ha sido muy productiva en la temática que nos unió, sin perder nuestra propia identidad de especialistas en cada campo profesional. Gracias doña Flor Garita Hernández por participarnos en estas décadas de su trabajo.

\section{Referencias bibliográficas}

Bedoya Benítez, E. (1994). Importancia de los nombres geográficos. Revista Geográfica, (29), 78-83.

Bedoya Benítez, E. (2007). Nombres geográficos. En J. P. Bergoeing y L. G. Brenes (Eds.), Prácticas de la Geografia (pp. 11-51). Cartago, Costa Rica: Editorial Tecnológica de Costa Rica. 
Chang, G. (2010). Toponimia de la provincia de Limón. San José, Costa Rica: Ministerio de Cultura y Juventud. Centro de Investigación y Conservación del patrimonio Cultural. Imprenta Nacional.

Garita, F. (1995). Toponimia de la Provincia de Cartago. San José, Costa Rica: Ministerio de Cultura Juventud y Deportes.

Garita, F. (1997). Toponimia de la provincia de Heredia. San José, Costa Rica: Instituto Geográfico Nacional.

Garita, F. (2000). Toponimia indígena de la provincia de Guanacaste. San José, Costa Rica: Instituto Geográfico Nacional.

Garita, F. (2002). Toponimia de la provincia de Puntarenas. San José, Costa Rica: Instituto Geográfico Nacional.

Garita, F. y Bedoya, E. (2010). Toponimia de la provincia de Alajuela. San José, Costa Rica: Editorial Universidad de Costa Rica.

Garita, F. y Bedoya, E. (2015). Toponimia de la red vial de la Gran Área Metropolitana. San José, Costa Rica: Editorial Universidad de Costa Rica.

Hudon, H. (1986). Metodología de inventarios toponímicos. Canadá: Documentación toponímica.

Monge, R., Bolaños, T., Fonseca M., Zumbado, M. y Guillén, A. (2003). Toponimia de la provincia de San José. San José, Costa Rica: Editorial Instituto Geográfico Nacional.

International Hydrographic Bureau (IHB). (1985). Standardization of Undersea feature names. Mónaco: Autor.

Morandi, M. C. (2001). Toponimia Antártica: La Tarea del Servicio de Hidrografía Naval en materia de imposición y resguardo en nombres geográficos en sus zonas de jurisdicción. Boletín del Servicio de Hidrografía Naval, 1(1), 197-202.

Ortiz, V. (1987). La naturaleza de los nombres geográficos y su origen lingüístico y cultural. México: Instituto Nacional de Estadística Geográfica e Informática. Dirección General de Geografía. 
Querol, E. (1995). La metodología en los estudios de toponimia. En Vicenç M. Rosselló y E. I. Casanova (Eds.), Materials de Toponímia (pp. 61-74). Valècia: Generalitat Valenciana - Universitat de Valècia.

Radal, R. (1986). Directorio de instituciones en las Américas involucradas. En Nombres geográficos. Mimeografiado. Publicación del Grupo de Trabajo de Gaceteros y Nombres Geográficos, Comisión de Cartografía, Instituto Panamericano de Geografía e Historia.

Rivas, D. (1986). Normalización de Nombres Geográficos. Comité Organizador de la II Reunión Regional de la División de la División de América Latina del Grupo de Expertos de la ONU. México.

Tort, J. Toponimia y marginalidad geográfica. Los nombres de lugar como reflejo de una interpretación del espacio. Scripta Nova. Revista electrónica de geografía y ciencias sociales, VII, (138). Recuperado de http://www.ub.es/geocrit/sn/sn138.html 